\title{
Association Between Hypertension, Body Mass Index, and Sleep: A Cross-Sectional Study
}

\author{
Aishwarya GonzalezCherubal, S Pooja, Vijaya Raghavan*
}

\begin{abstract}
Background: Sleep disorders can act as risk factors and even aggravate underlying conditions. With prevalence of $17 \%$ in general population, hypertension is a leading cause of morbidity and mortality in India. Though hypertension has various well established risk factors like family history, sedentary lifestyle, poor diet, smoking and age, sleep is often an understudied and overlooked factor. Body mass index is another important risk factor for various physical conditions. Associations between sleep and body mass index have been documented in many studies around the world. Although a consensus is yet to be drawn, many studies highlight that BMI related disorders could be predicted by sleep duration and quality.
\end{abstract}

Materials and Methods: Two hundred consecutive hypertensive patients who were attending the OPD for follow-up were included as participants in this study after obtaining an informed consent. A semi structured proforma was designed to elicit the socio demographic profile of the participants. Each participant was assessed for the presence of sleep disorders by sleep-50 questionnaire and quality of sleep by the Pittsburgh Sleep Quality Index (PSQI).

Results: Results found that BMI was significantly correlated with sleep quality, sleep duration, and sleep disorder. Hypertension was not significantly correlated to sleep quality or duration but associated to sleep disorder.

Conclusion: This study found that body mass index was significantly correlated with sleep variables such as sleep duration, sleep quality, and sleep disorders. Maintaining a healthy BMI could in fact impact the amount and quality of sleep an individual receives.

Keywords: Sleep, body mass index, hypertension, sleep disorders, cardiovascular disease
Running title: Relationship between hypertension, BMI, and sleep

Address for Correspondence: Dr. Vijaya Raghavan, Consultant Psychiatrist - Research, Schizophrenia Research Foundation, Anna Nagar West Extension, Chennai, India; E-mail: vijayaraghavan@scarfindia.org

How to Cite this Article: Cherubal AG, Pooja S, Vijaya Raghavan. Association between hypertension, body mass index, and sleep: A cross-sectional study. Indian Journal of Mental Health and NeuroSciences.2020;3(1):pp 11-14

$\mathrm{S}$ leep is important for the normal well-being of any individual and disturbances can lead to physical and psychological problems. The sleep cycle can alter autonomic nervous system functions that influence blood pressure and its related systems ${ }^{1}$. It is therefore a given that sleep disorders can affect a myriad of physiological problems such as hypertension, diabetes mellitus and cardiovascular disease. Sleep disorders can act as risk factors and even aggravate underlying conditions. This is a commonly reported phenomenon in hypertension. With prevalence of $17 \%$ in general population, hypertension is a leading cause of morbidity and mortality in India. Though hypertension has various well established risk factors like family history, sedentary lifestyle, poor diet, smoking and age, sleep is often an understudied and overlooked factor. Several studies in this regard have shown that there is a relationship between shorter duration of sleep and hypertension in adults. ${ }^{2}$

Obesity and hypertension are growing public health problems with expanding literature. In this context, body mass index is another important risk factor for various physical conditions. Associations between sleep and body mass index have been documented in many studies around the world. ${ }^{3}$ Although a consensus

${ }^{\star}$ Schizophrenia Research Foundation, R/7A, North Main Road, Anna Nagar West Extension, Chennai, Tamil Nadu, India 
is yet to be drawn, many studies highlight that BMI related disorders could be predicted by sleep duration and quality. In a similar way BMI could have a role to play in various sleep disorders.

Despite routine advice to keep sleep in check, studies report that individuals still suffer from a range of sleep related problems. A study conducted in India found that nearly $33 \%$ of people surveyed in the general population reported to be suffering from insomnia. ${ }^{4}$ Further research surrounding sleep and its association to physiological conditions is required to understand the nature of the relationship. Studies regarding the association between hypertension or BMI and sleep variables are limited in the Indian setup. Thus, the present study aims to describe the prevalence of sleep disorders in hypertensive individuals, quality of sleep and duration of sleep as compared with the normal population. This study also looks at finding correlations among the sleep variables and conditions like hypertension and BMI.

\section{MATERIAL \& METHODS}

The study was conducted in the Hypertension OPD, Department of General Medicine, Madras Medical College, Chennai. Two hundred consecutive hypertensive patients who were attending the OPD for follow-up were included as participants in this study after obtaining an informed consent. Patients were included in the study group if they were taking hypertension medication. The control group included non-hypertensive individuals who were patients at the clinic or relatives of patients. Participants with history of co-morbid medical and psychiatric illnesses were excluded from the study. A semi-structured proforma was designed to elicit the socio demographic profile of the participants. Each participant was assessed for the presence of sleep disorders by sleep-50 questionnaire and for quality of sleep by the Pittsburgh Sleep Quality Index (PSQI). Duration of sleep was asked directly to patients and recorded. Statistical analysis was carried out using analysis software SPSS version 20.

\section{RESULTS}

Participants' mean age from the sample was $49 \pm 12.52$. Among the 400 participants who were assessed in the study, 200 were hypertensive and 200 were normotensive. Out of these there were more normal ( $\mathrm{n}$ - 143) and overweight ( $\mathrm{n}$ - 35) participants in the normotensive group and more obese $(\mathrm{n}-38)$ in the hypertensive group. Results on sleep and hypertension found that hypertensive participants had poorer sleep quality (29.5\% vs $24 \%$ ), shorter sleep duration (23.5\% vs $19 \%$ ) and more sleep disorders (9.5\% vs 9, $4.5 \%$ ).

Table 1. Comparison of normotensive and hypertensive participants $(n=400)$

\begin{tabular}{|c|c|c|}
\hline Variable & $\begin{array}{c}\text { Normotensive (200) } \\
\text { N (\%) }\end{array}$ & $\begin{array}{c}\text { Hypertensive (200) } \\
\text { N (\%) }\end{array}$ \\
\hline $\begin{array}{l}\text { Sex } \\
\text { male } \\
\text { female }\end{array}$ & $\begin{array}{l}118(59 \%) \\
82(41 \%)\end{array}$ & $\begin{array}{l}118(59 \%) \\
82(41 \%)\end{array}$ \\
\hline $\begin{array}{l}\text { BMI } \\
\text { normal } \\
\text { overweight } \\
\text { obese }\end{array}$ & $\begin{array}{l}143(71.5 \%) \\
35(17.5 \%) \\
22(11 \%)\end{array}$ & $\begin{array}{l}132(66 \%) \\
30(15 \%) \\
38(19 \%)\end{array}$ \\
\hline $\begin{array}{l}\text { Sleep Quality } \\
\text { fair } \\
\text { poor }\end{array}$ & $\begin{array}{l}152(76 \%) \\
48(24 \%)\end{array}$ & $\begin{array}{l}141(70.5 \%) \\
59(29.5 \%)\end{array}$ \\
\hline $\begin{array}{l}\text { Sleep Duration } \\
>7 \text { hours } \\
<7 \text { hours }\end{array}$ & $\begin{array}{l}162(81 \%) \\
38(19 \%)\end{array}$ & $\begin{array}{l}153(76.5 \%) \\
47(23.5 \%)\end{array}$ \\
\hline $\begin{array}{l}\text { Sleep Disorder } \\
\text { No } \\
\text { yes }\end{array}$ & $\begin{array}{l}191(95.5 \%) \\
9(4.5 \%)\end{array}$ & $\begin{array}{l}181(90.5 \%) \\
19(9.5 \%)\end{array}$ \\
\hline
\end{tabular}

HAM-A - Hamilton rating scale for Anxiety; HAM-D - Hamilton rating scale for Depression 
Results found that BMI was significantly $(\mathrm{p}<0.01)$ correlated with sleep quality, sleep duration, and sleep disorder. Hypertension was not significantly correlated to sleep quality or duration but associated to sleep disorder $(<0.05)$ (table 2).

Table 2. Correlation between hypertension,BMI, and sleep disorders

\begin{tabular}{|c|c|c|c|}
\hline Variable & Sleep quality & Sleep duration & Sleep disorder \\
\hline \multirow{2}{*}{ BMI } & $-0.396^{* *}$ & $-0.213^{* *}$ & $0.317^{* *}$ \\
& $(0.000)$ & $(0.000)$ & $(0.000)$ \\
\hline \multirow{2}{*}{ Hypertension } & 0.062 & 0.055 & $-0.098^{*}$ \\
& $(0.215)$ & $(0.272)$ & $(0.050)$ \\
\hline
\end{tabular}

Statistical significance $-{ }^{*}{ }^{*} \mathrm{p}<0.01$ ( 2 tailed), ${ }^{\star} \mathrm{p}<0.05$ ( 2 tailed)

\section{DISCUSSION:}

The aim of the study was to describe the prevalence of sleep disorders, poor quality of sleep and shorter duration of sleep, in hypertensive individuals as compared to the normal population. A similar crosssectional investigation was made for body mass index.

This study found that more participants with hypertension had poorer sleep quality, shorter sleep duration and more sleep disorders than normotensive participants. These results are aligned with that of a review by Wang et al. where individuals whom reported less than seven hours of sleep had higher risk of hypertension. ${ }^{5}$ Furthermore, the review found that individuals reporting sleep for more than seven hours also posed a risk of hypertension. This study did not find any prevalence among hypertensive individuals and sleep longer than seven hours.

While this study reported a higher frequency of hypertensive participants with poorer quality, lesser duration and disordered sleep, there was no correlation between two of the three sleep variables with hypertension. Only sleep disorder was found to be negatively correlated to hypertension. Similarly studies have found that sleep disorders like insomnia were not associated with hypertension in older adults. ${ }^{6}$ One paper took into consideration short sleep duration as an effect of sleep disorder and found hypertension to be correlated. ${ }^{7}$ However, this study did not find duration of sleep to be significantly correlated.

Another major finding was that BMI was significantly correlated with the sleep variables. Results indicate that greater BMI is associated to lower quality of sleep and lesser duration of sleep. Our results are consistent with other studies that found a significant association between greater BMI and poor sleep duration among women and among samples of young and older adults. ${ }^{8,9}$ Conversely, a positive correlation was reported between BMI and sleep disorder. This relationship has been reported in numerous studies relating obesity with sleep apnea, insomnia, and restless leg syndrome. ${ }^{10}$ Such papers predict that exercise and weight loss may be effective in reducing risk of BMI related physical conditions.

\section{CONCLUSION}

This study found that body mass index was significantly correlated with sleep variables such as sleep duration, sleep quality, and sleep disorders. Maintaining a healthy BMI could in fact impact the amount and quality of sleep an individual receives. Although all sleep variables were incidentally higher among hypertensive as compared to normotensive patients, two of these variables were not found to be significantly correlated to hypertension. Literature supports these results as well as recommends that exercise be an intervention to reduce BMI and, consequentially, the risk of sleep related problems.

\section{REFERENCES}

1 CalhounDA \& HardingSM. Sleep and hypertension. Chest.2010;138(2):434-443

2 BansilP,Kuklina EV, Merritt RK et al. Associations between sleep disorders, sleep duration, quality of sleep, and hypertension: results from the National Health and Nutrition Examination Survey, 2005 to 2008. Journal of Clinical Hypertension. 2011;13(10):739-743 
3 Grandner MA, Schopfer EA, Sands-LincolnM, et al. The Relationship between Sleep Duration and Body Mass Index Depends on Age. Obesity.2015;23(12): 2491-2498

4 BhaskarS, Hemavathy D \& Prasad S. Prevalence of chronic insomnia in adult patients and its correlation with medical comorbidities. Journal of Family Medicine and Primary Care. 2015;5(4): 780-784

5 Wang Y, Mei H, Jiang YR., et al. Relationship between Duration of Sleep and Hypertension in Adults: A Meta-Analysis. Journal of Clinical Sleep Medicine. 2015; 11(9): 1047-1056

6 Phillips B, Bůzková P, Enright P, \& Cardiovascular Health Study Research Group. Insomnia did not predict incident hypertension in older adults in the cardiovascular health study. Sleep. 2009;32(1): 65-72

7 Vgontzas AN, Liao D, Bixler EO, et al. Insomnia with objective short sleep duration is associated with a high risk for hypertension. Sleep. 2009;32(4):491-497

8 KrističevićT, Štefan L, \&Sporiš G. The Associations between Sleep Duration and Sleep Quality with BodyMass Index in a Large Sample of Young Adults. International Journal of Environmental Research and Public Health. 2018;15(4):758

9 Sasaki N, Fujiwara S, Yamashita H, et al. Association between obesity and self-reported sleep duration variability, sleep timing, and age in the Japanese population. Obesity Research and Clinical Practice. 2018;12(2):187-194

10 Romero-Corral A, Caples SM, Lopez-Jimenez F, et al. Interactions Between Obesity and Obstructive Sleep Apnea. Chest.2010;137(3):711-719 